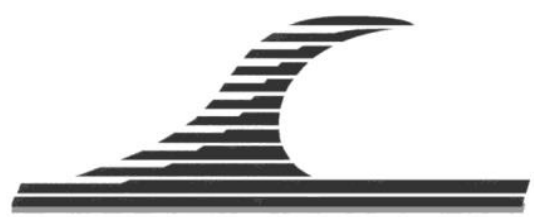
Jarlan.

(C) Editions Paralia CFL

\title{
Conception d'ouvrages côtiers verticaux en enrochements
}

\author{
Paul MILESI ${ }^{1}$, Olivier KIMMOUN ${ }^{2}$, David LAJOIE ${ }^{1}$
}

1. SAS Hydro GC, 772 chemin du Flaquier Nord 06530 Le Tignet, France. paul.milesi@hydrogc.fr

2. Ecole Centrale Marseille, 38 rue Frédéric Joliot-Curie13013 Marseille, France. olivier.kimmoun@centrale-marseille.fr

\section{Résumé :}

Les ouvrages verticaux en enrochements sont des dispositifs innovants et alternatifs aux ouvrages de type caissons verticaux. Les blocs sont encagés par une structure métallique ou des pieux, le tout occupant une emprise réduite au sol. Ce type d'ouvrage offre de multiples avantages : impact faible en cours de travaux, possibilité de s'implanter à proximité des zones de vie marine sans impact significatif, perméabilité laissant circuler une partie des courants. Dans le cadre du réaménagement du port de Banyuls-sur-Mer, la construction d'un musoir en enrochements verticalisé de $20 \mathrm{~m}$ de diamètre permet d'améliorer significativement la qualité du bassin portuaire et de respecter les herbiers environnants. Un modèle numérique de diffraction 2D/3D aux équations intégrales a été développé de manière à évaluer, sans calcul lourd, différentes configurations d'ouvrages poreux, pouvant associer par exemple caissons Jarlan et enrochements. Des premiers essais en modèle réduit physique ont permis de confirmer le fonctionnent d'ouvrages poreux simples. Une seconde campagne expérimentale a permis de tester une première géométrie d'ouvrages innovants.

Article issu d'une sélection des XVèmes Journées Nationales Génie Côtier Génie Civil qui se sont tenues à La Rochelle du 29 au 31 mai 2018.

Soumis le 13 juin 2019, accepté le 23 octobre 2021, en ligne le 22 novembre 2021.

La seule version examinée est celle écrite en français. La ou les autres versions n'étant pas examinées par le comité de rédaction de la revue, sont donc publiées sous l'entière responsabilité du ou des auteurs.

A SUORT TRANSLATED VERSION IN ENGLISH IS AVAILABLE ONLINE

Pour citer cet article :

MILESI P., KIMMOUN O., LAJOIE D. (2021). Conception d'ouvrages côtiers verticaux en enrochements. Revue Paralia, Vol. 14, pp s01.1-s01.12.

DOI: https://doi.org/10.5150/revue-paralia.2021.s01 


\section{Introduction}

Les études de conception de nouveaux ouvrages maritimes en zone littorale française sont généralement menées dans le cadre d'aménagements de ports existants. L'extension des surfaces de bassins et l'amélioration de l'agitation constituent les axes principaux de développement des ports de plaisance en justifiant bien souvent un investissement en termes d'infrastructures. C'est par exemple le cas du réaménagement du port de plaisance de Banyuls-sur-Mer (66), qui est l'occasion d'améliorer significativement la qualité de son bassin portuaire par, entre autres, l'allongement de la digue principale.

L'extension d'ouvrages en mer implique depuis de nombreuses années le devoir du respect de l'environnement. Les digues à talus, très larges, construites au début du XXème siècle pour délimiter les bassins portuaires en eaux profondes ont été progressivement remplacées par des ouvrages de type caissons verticaux plus économiques et moins consommateurs de matériaux de carrière. L'empiétement sur les fonds marins est nettement plus réduit. D'autres alternatives aux caissons verticaux amortisseurs en béton ont été imaginées, comme des ouvrages verticaux en enrochements. Les blocs sont maintenus par une structure métallique ou des pieux. Ce type d'ouvrage offre la possibilité de s'implanter à proximité des zones de vie marine sans impact significatif.

A Banyuls-sur-Mer, la construction d'un musoir en enrochements verticalisés de $20 \mathrm{~m}$ de diamètre dans une charpente métallique permettrait d'enrayer les problèmes d'agitation portuaire aujourd'hui rencontrés avec les houles de tempêtes de provenance Sud-Est (figure 1). Les zones d'herbiers protégés sont par ce procédé maintenues à au moins $5 \mathrm{~m}$ du pied de la structure.

Un travail de recherche a été entrepris pour modéliser le comportement d'ouvrages poreux innovants en enrochements, face à la houle.

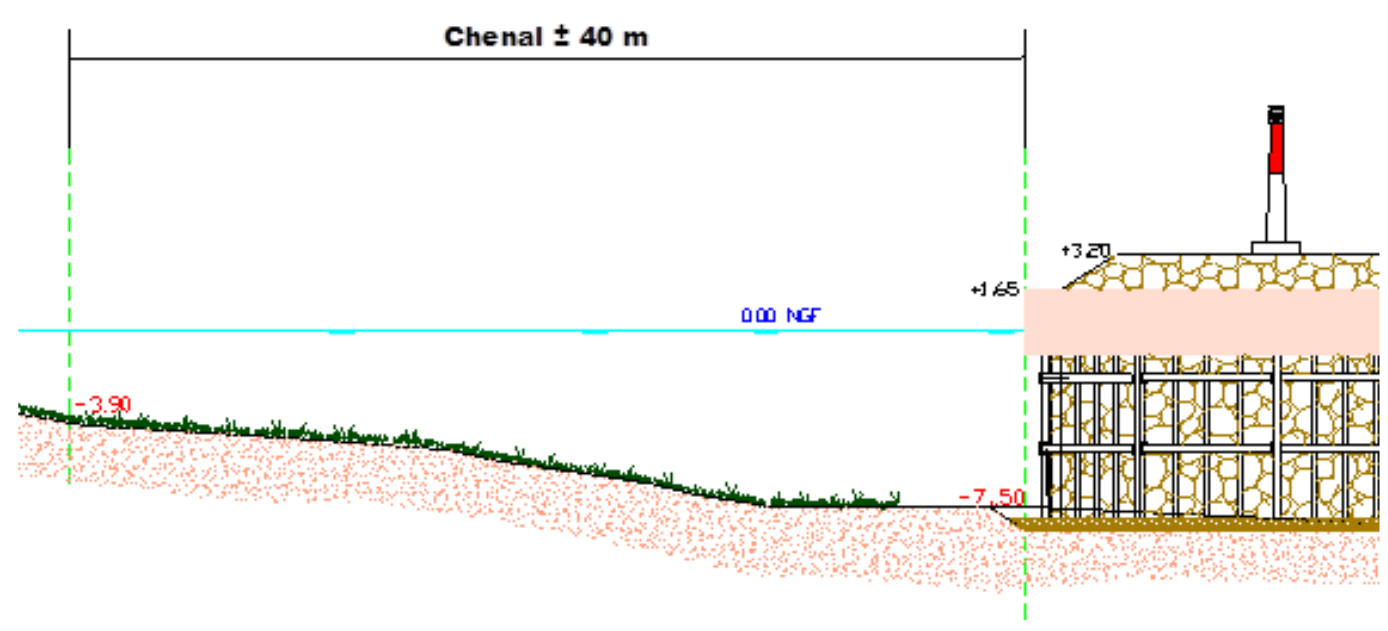

Figure 1. Configuration du musoir verticalisé. 


\section{Configuration expérimentale : Ouvrage poreux simple}

Deux séries d'essais ont été réalisées dans le canal à houle de l'Ecole Centrale de Marseille, d'une longueur utile d'environ $17 \mathrm{~m}$ par $60 \mathrm{~cm}$ de large et muni d'un batteur de type "volet". Une plage-amortisseur est disposée en bout de canal. La première campagne expérimentale a eu pour but d'explorer le fonctionnement d'un ouvrage poreux simple en enrochements en mesurant la réflexion et la transmission. Une seconde campagne présentée au chapitre 6 teste une géométrie innovante d'ouvrages poreux à gabions espacés.

La similitude de Froude à l'échelle $1 / 10^{\text {ème }}$ permet de reproduire les forces appliquées au fluide dont l'écoulement est turbulent dans le milieu poreux. La maquette (figure 2) est une cage avec une structure en acier permettant de contenir les blocs qui assureront la dissipation de l'énergie de la houle incidente. La largeur du dispositif en enrochements est de $24 \mathrm{~cm}$. La hauteur d'eau est de $20 \mathrm{~cm}$.
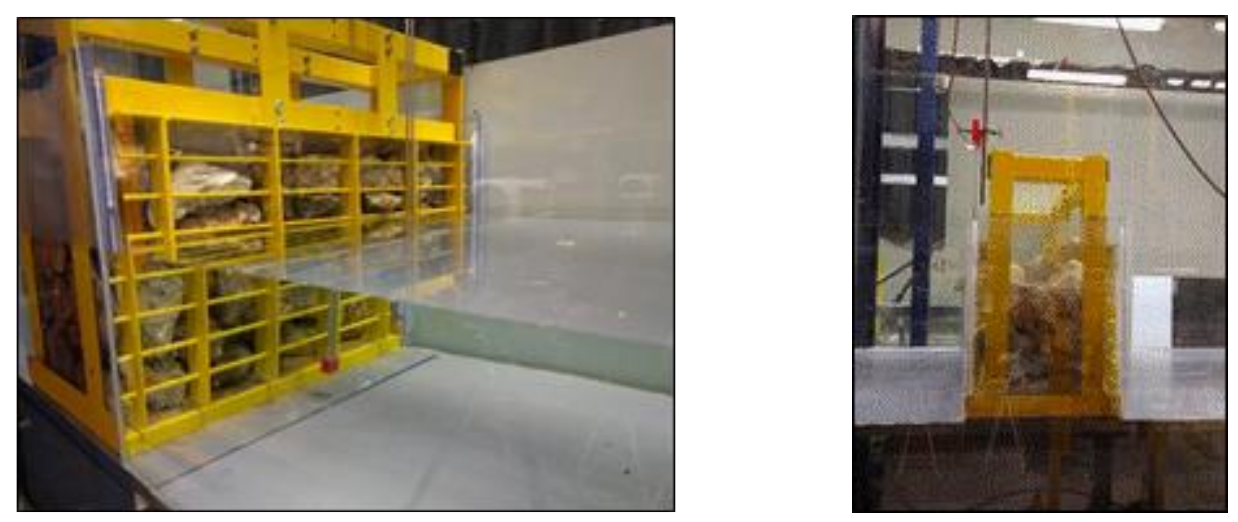

Figure 2. Vues oblique et latérale de la maquette.

Les enrochements sélectionnés pour la campagne d'essais sont des enrochements de type calcaire dont la masse volumique moyenne d'un échantillon type a été mesurée à $\rho \approx 2620 \mathrm{~kg} \mathrm{~m}^{-3}$. Dans un premier temps, trois granulométries types ont été sélectionnées dans le but d'explorer l'influence de la taille des enrochements et du taux de porosité sur les caractéristiques hydrodynamiques de l'ouvrage. Les enrochements ont été pesés un par un avant chaque série d'essais afin de déterminer la courbe granulométrique correspondante à l'échantillon en place ainsi que pour déterminer la porosité. Les granulométries types utilisées dans la campagne d'essais sont les suivantes :

Tableau 1. Caractéristiques des granulométries.

\begin{tabular}{ccccccc}
\hline $\begin{array}{c}\text { Type de } \\
\text { granulométrie }\end{array}$ & $\boldsymbol{M}_{\mathbf{1 0}}$ & $\boldsymbol{M}_{\boldsymbol{5 0} \boldsymbol{0}}$ & $\boldsymbol{M}_{\boldsymbol{8 5}}$ & $\boldsymbol{D}_{\boldsymbol{n} \boldsymbol{5 0}}$ & $\boldsymbol{n}_{\boldsymbol{R} \boldsymbol{R} \boldsymbol{D}}$ & Porosité $\boldsymbol{n}$ \\
\hline $\mathrm{G} 1$ & $170 \mathrm{~g}$ & $233 \mathrm{~g}$ & $282 \mathrm{~g}$ & $4.5 \mathrm{~cm}$ & 17.2 & $41.5 \%$ \\
$\mathrm{G} 2$ & $527 \mathrm{~g}$ & $617 \mathrm{~g}$ & $716 \mathrm{~g}$ & $6.2 \mathrm{~cm}$ & 28.4 & $43.6 \%$ \\
$\mathrm{G} 3$ & $1204 \mathrm{~g}$ & $1861 \mathrm{~g}$ & $2647 \mathrm{~g}$ & $8.9 \mathrm{~cm}$ & 11.0 & $44.8 \%$ \\
\hline
\end{tabular}


On remarque que l'évolution nette de la masse médiane $\mathrm{M}_{50}$ entre les granulométries types induit une évolution très légère du taux de porosité correspondante. L'étalement des taux de porosité est au final assez restreint avec $41.5 \% \leq n \leq 44.8 \%$. Ils se trouvent dans la tranche haute des taux de porosité réalistes définies par VAN GENT (1995), $35 \% \leq n \leq 45 \%$. La contrainte des parois de la cage comparée à un empilement "libre" des enrochements pourrait expliquer ces porosités élevées comme proposé par MELLINK (2012). La disposition des enrochements lors de leur mise en place a aussi eu un impact, au maximum $2 \%$, sur les taux de porosité calculés.

\section{Résultats expérimentaux de la $1^{\text {ère }}$ campagne}

Les coefficients de réflexion et de transmission en houles régulières sont calculés via une méthode de séparation développée par ANDERSEN et al. (2017). Deux séries de cinq sondes limnométriques étaient disposées de part et d'autre de la maquette. Les incertitudes de mesure résident principalement dans le calibrage des sondes.

La performance hydrodynamique d'un ouvrage à dissiper la houle incidente peut être synthétisée par le calcul de l'énergie dissipée $E_{d}$ :

$$
E_{d}=1-\left(k_{r}^{2}+k_{t}^{2}\right)
$$

$k_{r}$ et $k_{t}$ sont respectivement les coefficients de réflexion et de transmission.

L'énergie dissipée $E_{d}$ par l'ouvrage poreux est nettement supérieure pour les petites périodes que pour les grandes. Elle diminue logiquement au fur et à mesure que les longueurs d'onde augmentent devant la largeur de l'ouvrage. La performance de l'ouvrage est aussi meilleure face aux grandes amplitudes. Ceci est expliqué par la présence de vitesses plus importantes et par une plus grande dissipation par le terme de frottement turbulent en $v^{2}$. Les courbes ci-dessous (figure 3) présentent les résultats des performances hydrodynamiques pour la hauteur de houle incidente $\mathrm{a}_{\mathrm{i}}=2.5 \mathrm{~m}$.
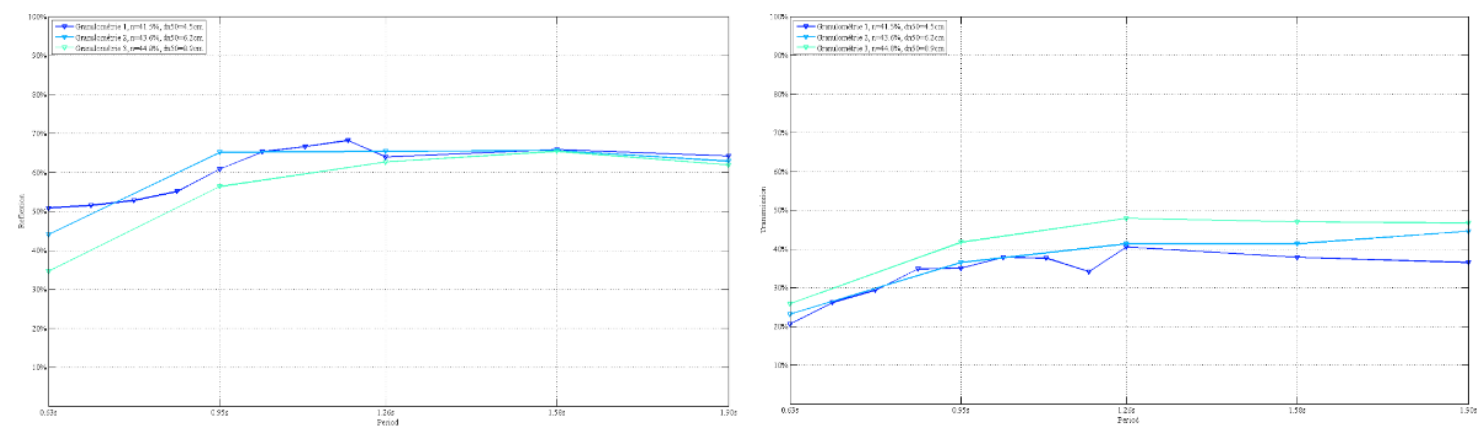

Figure 3. Comparaison des granulométries 1,2 et $3, a_{i}=1.25 \mathrm{~cm}$.

\section{Modélisation d'un écoulement engendré par la houle dans un milieu poreux}

On considère un volume fermé contenant le milieu poreux. La conservation de la quantité de mouvement à l'intérieur du domaine a pour expression : 


$$
\iiint_{\mathrm{F}}\left(\frac{\partial \overrightarrow{\mathrm{v}}}{\partial \mathrm{t}}+\overrightarrow{\mathrm{v}} \cdot \overrightarrow{\operatorname{grad}} \overrightarrow{\mathrm{v}}\right) \mathrm{dV}=-\frac{1}{\rho} \iint_{\partial \Sigma} \mathrm{p} \overrightarrow{\mathrm{dS}}+\iiint_{\Sigma} \overrightarrow{\mathrm{f}_{\mathrm{r}}} \mathrm{dV}+\iiint_{\mathrm{F}} \overrightarrow{\mathrm{g}} \mathrm{dV}
$$

où : $\overrightarrow{\mathrm{f}_{\mathrm{r}}}$ sont les forces résistantes à l'échelle microscopique, $\sum$ représente le domaine total (poreux et fluide), $\mathrm{F}$ le domaine fluide, $\partial \sum$ la surface extérieure du domaine $\sum$, et $\overrightarrow{\mathrm{v}}$ la vitesse d'infiltration. A l'échelle macroscopique, pour un écoulement non stationnaire dans un milieu poreux, les forces de frottement peuvent être modélisées par l'équation de FORCHHEIMER (1901) étendue par POLUBARINOVA-KOCHINA (1952) qui a ajouté un terme d'inertie :

$$
\overrightarrow{\mathrm{F}_{\mathrm{r}}}=-\left[a \overrightarrow{v_{d}}+\mathrm{b}\left\|\overrightarrow{\mathrm{v}_{\mathrm{d}}}\right\| \overrightarrow{\mathrm{v}_{\mathrm{d}}}+\mathrm{c} \frac{\partial \overrightarrow{\mathrm{v}_{\mathrm{d}}}}{\partial \mathrm{t}}\right]
$$

$\mathrm{a}$, b et c sont des coefficients propres au milieu poreux considéré, $\overrightarrow{\mathrm{v}_{\mathrm{d}}}$ est la vitesse de flux (Darcy) définie par $\vec{v}_{d}=n \vec{v}$, où n est le taux de porosité. Les coefficients de frottement retenus sont ceux de BURCHARTH et ANDERSEN (1995) :

$$
\mathrm{a}=\alpha \frac{(1-\mathrm{n})^{2}}{\mathrm{n}^{3}} \frac{\mathrm{v}}{\mathrm{d}_{\mathrm{n} 50}^{2}} \quad \mathrm{~b}=\beta \frac{1-\mathrm{n}}{\mathrm{n}^{3}} \frac{1}{\mathrm{~d}_{50}} \quad \mathrm{c}=\frac{(1-\mathrm{n})}{\mathrm{n}} \mathrm{C}_{\mathrm{M}}
$$

où : $d_{n 50}$ est le diamètre nominal médian des blocs, $\alpha$ et $\beta$ sont des coefficients de tortuosité, $C_{M}$ est le coefficient d'inertie. L'équation (2) peut être réécrite à l'échelle macroscopique en introduisant la vitesse de flux $\vec{v}_{d}$ :

$$
\mathrm{S} \frac{\partial \overrightarrow{\mathrm{v}}_{\mathrm{d}}}{\partial \mathrm{t}}+\frac{1}{2 \mathrm{n}^{2}} \overrightarrow{\operatorname{grad}} \overrightarrow{\mathrm{v}}_{\mathrm{d}}^{2}=-\frac{1}{\rho} \overrightarrow{\operatorname{grad}} \mathrm{p}-\mathrm{g} \overrightarrow{\mathrm{z}}-\frac{v}{\mathrm{~K}} \overrightarrow{\mathrm{v}}_{\mathrm{d}}-\frac{\mathrm{C}_{\mathrm{f}}}{\sqrt{\mathrm{K}}}\left\|\overrightarrow{\mathrm{v}_{\mathrm{d}}}\right\| \overrightarrow{\mathrm{v}_{\mathrm{d}}}
$$

où les relations entre les coefficients $(\mathrm{a}, \mathrm{b}, \mathrm{c})$ et les coefficients de frottement $\left(\mathrm{K}, \mathrm{C}_{\mathrm{f}}\right)$ et d'inertie S (SOLLIT \& CROSS, 1972) sont :

$$
\mathrm{K}=\frac{\mathrm{v}}{\mathrm{a}} \quad \mathrm{C}_{\mathrm{f}}=\mathrm{b} \sqrt{\mathrm{K}} \quad \mathrm{S}=1+\mathrm{c}
$$

On linéarise l'équation (5) en utilisant la procédure de LORENTZ qui consiste à remplacer le terme de dissipation par une expression linéaire, en introduisant un coefficient de dissipation $f \omega$ adimensionnel tel que :

$$
\mathrm{f} \omega=\frac{v}{\mathrm{~K}}+\frac{\mathrm{C}_{\mathrm{f}}}{\sqrt{\mathrm{K}}} \overline{\mathrm{V}_{\mathrm{d}}}
$$

où :

$$
\overline{\mathrm{v}_{\mathrm{d}}}=\iiint_{\mathrm{P}} \frac{\int_{0}^{\mathrm{T}}\left|\overrightarrow{\mathrm{v}_{\mathrm{d}}}\right|^{3} \mathrm{dt}}{\int_{0}^{\mathrm{T}}\left|\overrightarrow{\mathrm{v}_{\mathrm{d}}}\right|^{2} \mathrm{dt}}
$$

L'écoulement dans le milieu continu fluide externe au milieu poreux est supposé irrotationnel, d'où l'existence d'un potentiel des vitesses $\Phi_{\mathrm{F}}$. En homogénéisant le milieu poreux, on le considère comme un milieu continu et on y introduit aussi un potentiel des vitesses $\Phi_{\mathrm{p}}$. En négligeant le terme quadratique en $\overrightarrow{\operatorname{grad}} \vec{v}_{\mathrm{d}}^{2}$ et en remplaçant $\vec{v}_{\mathrm{d}}$ par $\overrightarrow{\operatorname{grad}} \Phi_{\mathrm{p}}$, on aboutit à :

$$
\mathrm{S} \frac{\partial \Phi_{\mathrm{p}}}{\partial \mathrm{t}}+\frac{\mathrm{p}_{\mathrm{p}}}{\rho}+\mathrm{gz}+\mathrm{f} \omega \Phi_{\mathrm{p}}=0
$$




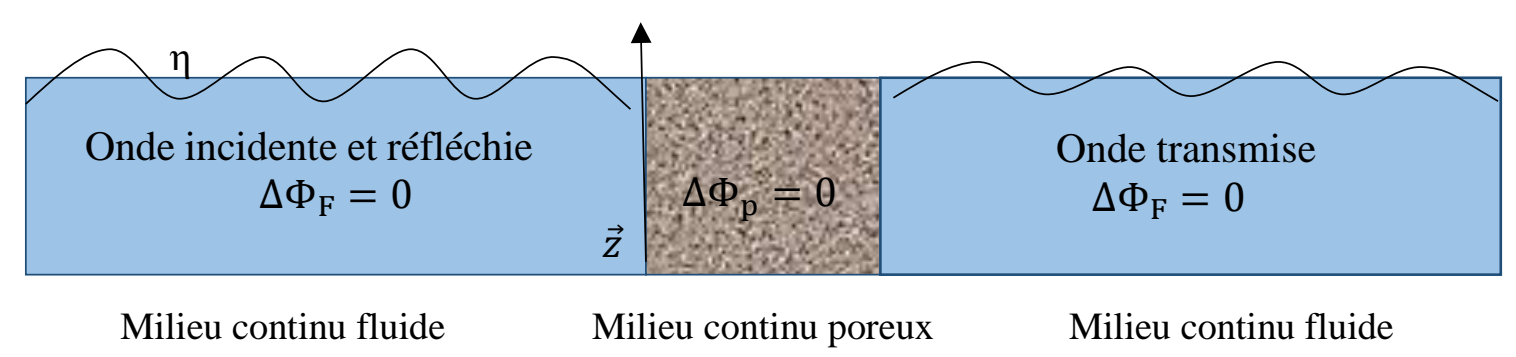

Figure 4. Configuration théorique et expérimentale.

La condition de surface libre doit respecter les conditions dynamiques et cinématiques données respectivement par :

$$
\begin{aligned}
& (\mathrm{f}-\mathrm{iS}) \omega \Phi_{\mathrm{p}}+\mathrm{g} \eta=0 \text { en } \mathrm{z}=\eta \\
& \mathrm{n} \frac{\mathrm{d} \eta}{\mathrm{dt}}=\frac{\mathrm{dz} \text { particule }}{\mathrm{dt}} \text { en } \mathrm{z}=\eta
\end{aligned}
$$

Le concept de porosité surfacique en opposition à la porosité volumique est introduit et défini par $\mu=1-(1-n)^{\frac{2}{3}}$. Après projection de l'équation (11) sur le système d'axes cartésiens, et après linéarisation, on obtient :

$$
-i \omega \eta=\frac{\mu}{n} \frac{\partial \Phi_{p}}{\partial z} \text { en } z=\eta
$$

La condition de surface libre à l'intérieur d'un milieu poreux est trouvée après la combinaison des équations (10) et (12) :

$$
\frac{\partial \Phi_{\mathrm{p}}}{\partial \mathrm{z}}-(\mathrm{S}+\mathrm{if}) \frac{\omega^{2}}{g} \frac{n^{2}}{\mu} \Phi_{\mathrm{p}} \text { en } \mathrm{z}=\eta
$$

L'interface entre deux milieux continus 1 et 2 doit respecter la conservation du flux et la continuité des pressions, exprimées respectivement par :

$$
\begin{aligned}
& \frac{\mu_{1}}{\mathrm{n}_{1}} \frac{\partial \Phi_{\mathrm{p} 1}}{\partial \mathrm{t}}=\frac{\mu_{2}}{\mathrm{n}_{2}} \frac{\partial \Phi_{\mathrm{p} 2}}{\partial \mathrm{t}} \text { à l'interface } \\
& \left(\mathrm{S}_{1}+\mathrm{if}_{1}\right) \Phi_{\mathrm{p} 1}=\left(\mathrm{S}_{2}+\mathrm{if}_{2}\right) \Phi_{\mathrm{p} 2} \text { à l'interface }
\end{aligned}
$$

\section{Résultats numériques de la $1^{\text {ère }}$ campagne}

Le système harmonique est résolu par une méthode de singularités sur la base d'une distribution mixte de sources et de doublets de RANKINE. On assure la continuité des flux et des pressions à travers les différents milieux fluides ou poreux. La non linéarité présente dans le terme $\mathrm{f}\left(\overrightarrow{\mathrm{v}_{\mathrm{d}}}\right)$ est calculée par itérations successives jusqu'à convergence. Les résultats des essais et des simulations correspondantes pour une hauteur de houle $\mathrm{a}_{\mathrm{i}}=25 \mathrm{~cm}$ à l'échelle réelle sont reportés ci-dessous (figure $5:$ Granulométrie 1, 2 et 3). L'erreur quadratique moyenne relative (RMSE) est donnée à titre indicatif.

Les résultats $\mathrm{du}$ modèle numérique vis-à-vis des données expérimentales sont satisfaisants avec toutefois une sous-estimation de la transmission pour les petits blocs. 


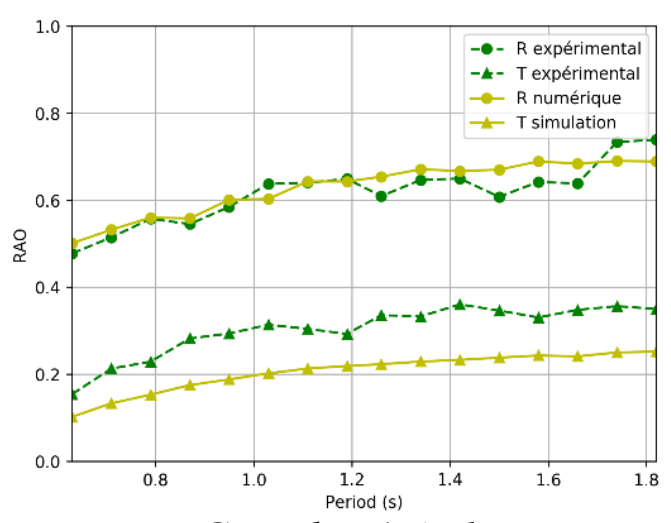

Granulométrie 1

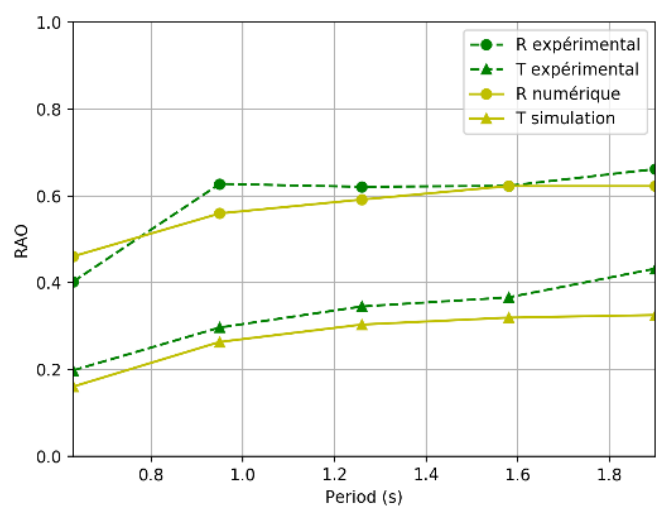

Granulométrie 2

RMSE $R=0.052$, RMSE $T=0.302$

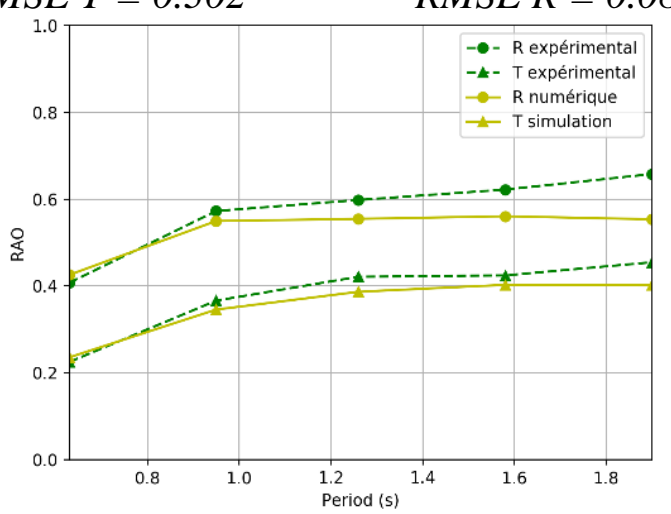

Granulométrie 3

RMSE $R=0.110$, RMSE $T=0.077$

Figure 5. Courbes de résultats, ai=2.5m, granulométrie $G 2$.

\section{Seconde campagne expérimentale : Géométrie innovante}

La conception d'un caisson Jarlan à l'aide d'un modèle numérique de diffraction est rendue possible depuis que l'équation (17) fut développée par Bougis et Lajoie (LAJOIE, 2008).

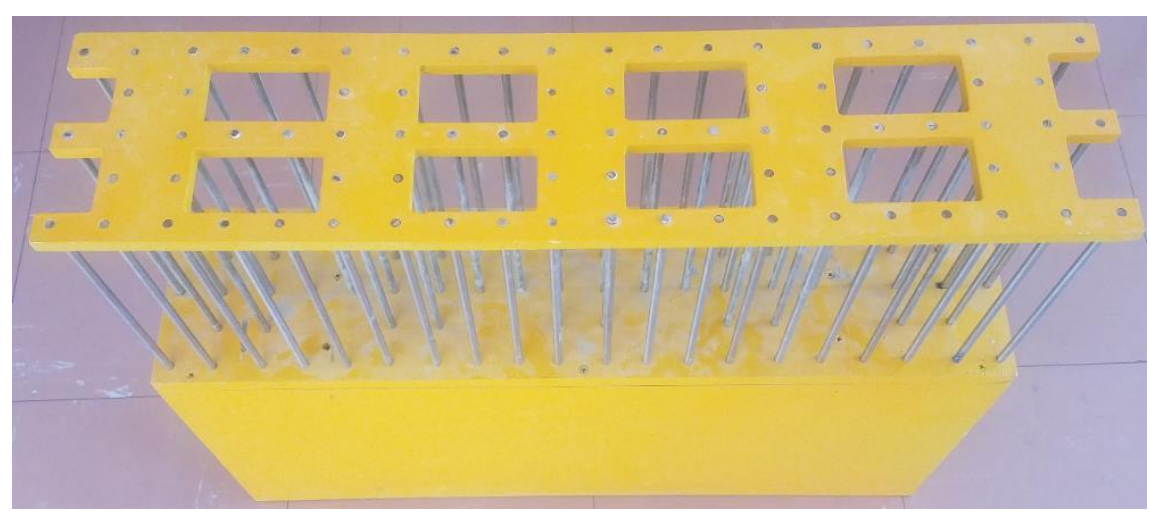

Figure 6. Vue oblique de la maquette.

L'idée innovante consiste à créer un ouvrage amortisseur à l'aide de plusieurs gabions espacés de manière à obtenir in fine les caractéristiques d'amortissement d'un caisson 
Jarlan, en réduisant drastiquement les quantités de béton. Les milieux poreux sont par ailleurs favorables aux juvéniles et leur poids permet d'apporter du lest pour la stabilité global de l'ouvrage.

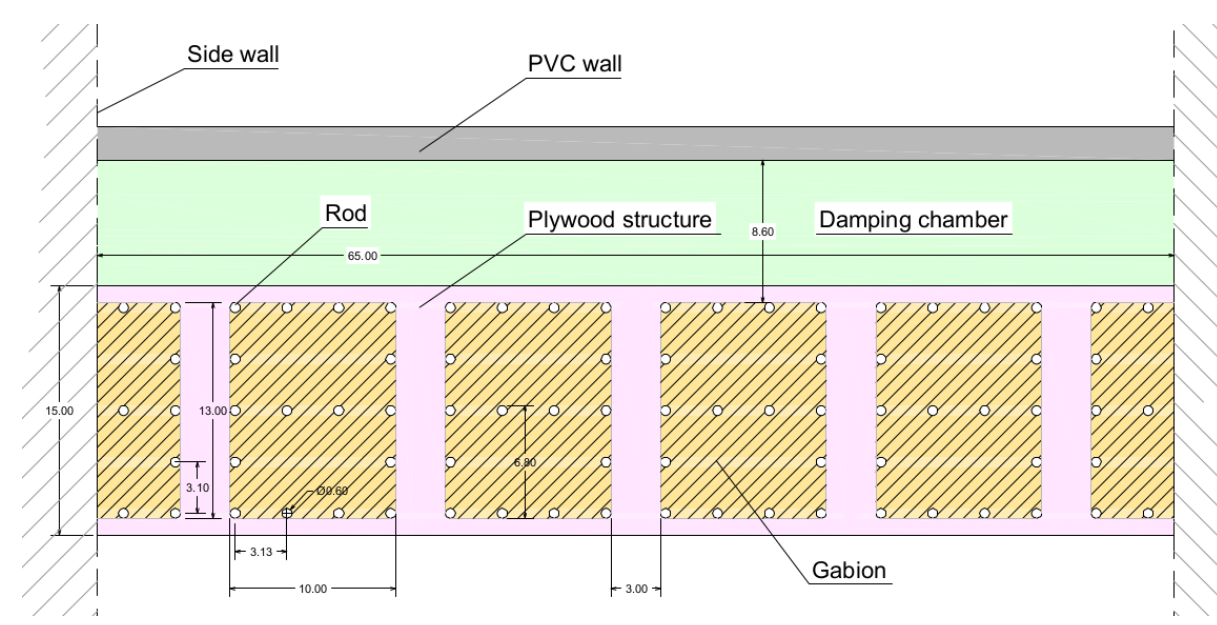

Figure 7. Schéma, vue du dessus.

La maquette de ce dispositif est représentée par les figures 6 et 7. Les cailloux qui constituent les gabions sont maintenus en place par un réseau de cylindres. Les trous sur la partie supérieure permettent de les disposer. Le modèle réduit contient ainsi 5 largeurs de gabions pour 5 fentes. La porosité de l'ouvrage sans tenir compte de la porosité des gabions, est de $\boldsymbol{\tau}=\mathbf{2 3} \%$. Une plaque est installée à l'arrière pour modéliser le fond de l'ouvrage. La chambre d'expansion mesure $8.6 \mathrm{~cm}$. Les gabions sont relevés de $30 \mathrm{~cm}$. La hauteur d'eau est de $15.6 \mathrm{~cm}$ à l'intérieur de la structure. Les caractéristiques des cailloux utilisés pour former les gabions sont reportées dans le tableau 2.

Tableau 2. Caractéristiques des granulométries.

\begin{tabular}{cccccc}
\hline$M_{15}$ & $M_{50}$ & $M_{85}$ & $D_{\boldsymbol{n} 50}$ & $\boldsymbol{n}_{\boldsymbol{R} \boldsymbol{D} \boldsymbol{D}}$ & Porosité $\boldsymbol{n}$ \\
\hline $25 \mathrm{~g}$ & $40 \mathrm{~g}$ & $53 \mathrm{~g}$ & $2.7 \mathrm{~cm}$ & 18.3 & $41 \%$ \\
\hline
\end{tabular}

\section{Principes de modélisation d'une paroi opaque perforée}

La pénétration d'un courant à travers une paroi opaque perforée est traitée de manière différente que pour un milieu poreux. Le gradient de pression des deux côtés de la paroi (figure 8) peut être exprimé via l'intégration de l'équation de Bernouilli-Lagrange le long d'une ligne de courant passant au travers d'un des trous (LAJOIE, 2008). Les termes de l'équation (16) correspondent aux pertes de pressions singulières et linéaires.

$$
\Delta p=\frac{1}{2} \rho K\left|\mathrm{v}_{\mathrm{d}}\right| \mathrm{v}_{\mathrm{d}}+\int_{0}^{a l} \rho \frac{1-\tau}{\tau k_{c}} \frac{\partial \mathrm{v}_{\mathrm{d}}}{\partial t} \cdot d l
$$

$\mathbf{v}_{\mathbf{d}}$ est la vitesse moyenne de l'écoulement à l'amont ou à l'aval des perforations ;

$\boldsymbol{\tau}$ est le taux de porosité ; 
$\boldsymbol{k}_{\boldsymbol{c}}$ est le coefficient de striction de section lors du passage d'un jet dans une ouverture ; $\boldsymbol{K}$ est le coefficient de pertes de charge à travers la plaque perforée, dont une expression appropriée est $\frac{1-\varepsilon}{\varepsilon^{2} k_{c}^{2}}$.

$a l=l+l m . l$ est l'épaisseur de la paroi perforée et $l m$ un petit terme correspondant aux effets de masse ajoutée.

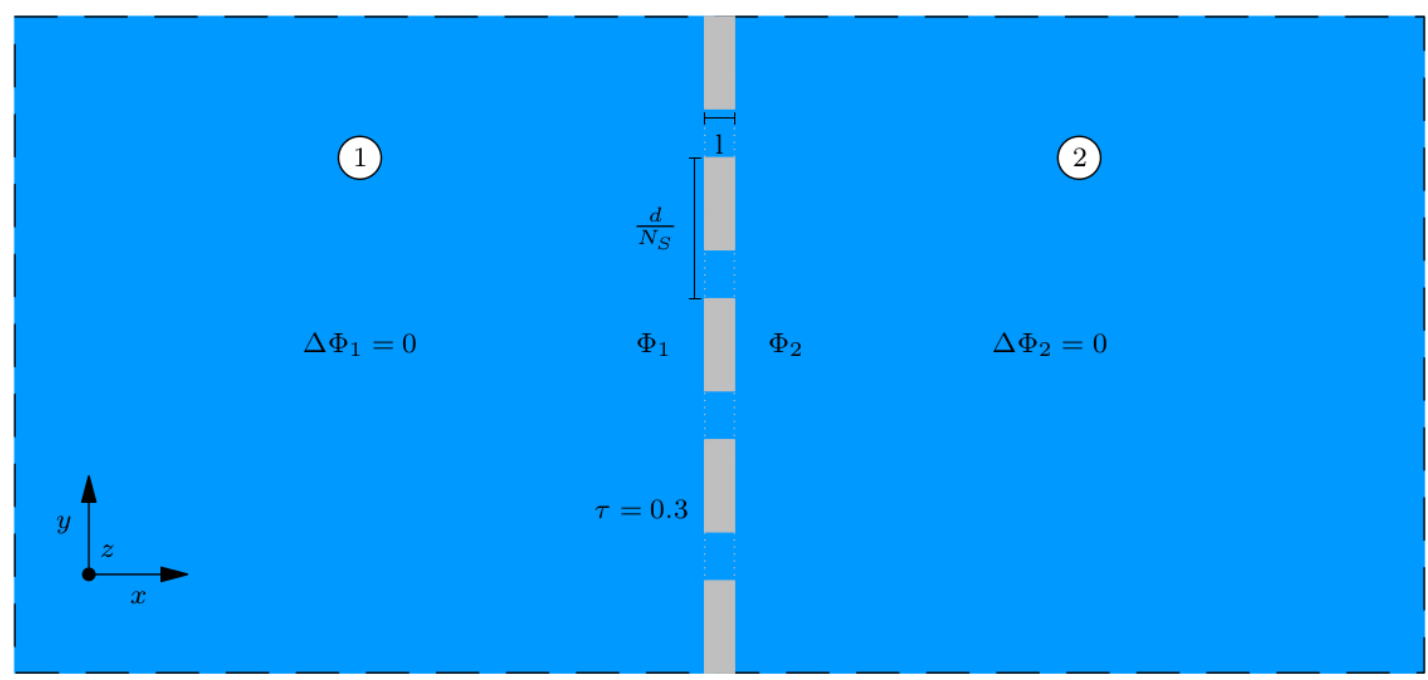

Figure 8. Principe de modélisation autour d'une paroi poreuse.

En retenant l'hypothèse des ondes monochromatiques, et après linéarisation de Lorentz, on retrouve l'équation développée par Bougis et Lajoie (LAJOIE, 2008) à partir de l'équation (16), mais étendue aux milieux poreux, reliant le potentiel complexe des vitesses $\phi_{1}$ et $\phi_{2}$ de part et d'autre de la paroi perforée, à la vitesse normale moyenne à travers la paroi :

$$
\frac{\partial \phi}{\partial n}=\frac{\varepsilon_{2} \boldsymbol{\phi}_{2}-\varepsilon_{1} \boldsymbol{\phi}_{1}}{\frac{a_{l}(1-\tau)}{\tau k_{c}} \frac{\mu_{1}}{n_{1}}+\frac{i}{\sqrt{2}} \sqrt{-\left(\frac{a_{l}(1-\tau)}{\tau k_{c}} \frac{\mu_{1}}{n_{1}}\right)^{2}+\sqrt{\left(\frac{a_{l}(1-\tau)}{\tau k_{c}} \frac{\mu_{1}}{n_{1}}\right)^{4}+4\left(\frac{4 K}{3 \pi \omega} \frac{\mu_{1}^{2}}{n_{1}^{2}}\right)^{2}\left|\varepsilon_{2} \boldsymbol{\phi}_{2}-\varepsilon_{1} \boldsymbol{\phi}_{1}\right|^{2}}}}
$$

$\varepsilon_{j}=S_{j}+i f_{j}, j \in[1,2]$

Le code numérique de diffraction permet la modélisation d'une paroi perforée de deux manières différentes : la paroi est modélisée en 2D via la formulation théorique (17), la paroi est modélisée en 3D en maillant les fentes sur toute leur épaisseur.

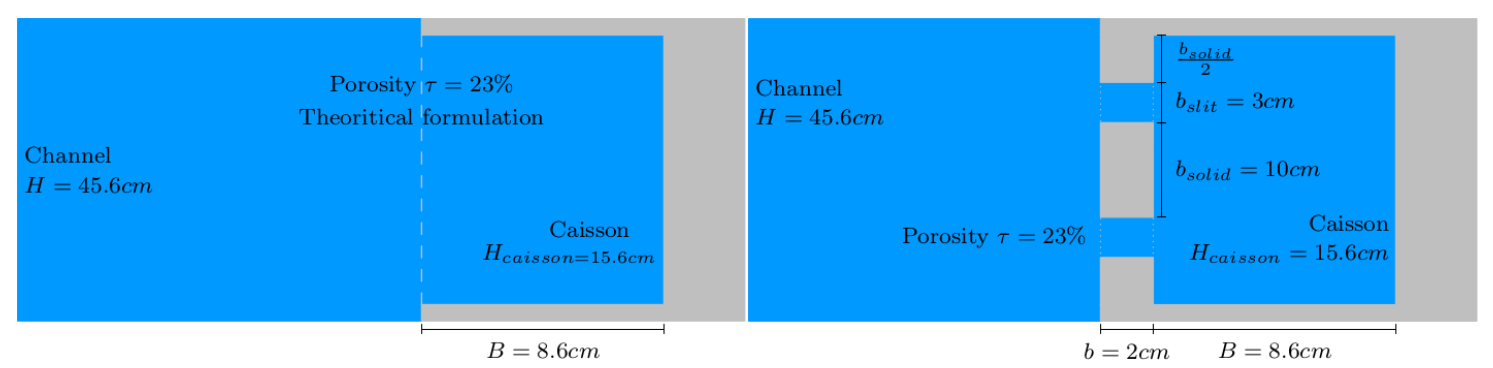

Figure 9. Vue du haut :Modèle 2D et modèle 3D. 
Des résultats numériques similaires sont recherchés entre les deux modèles. Dans la modélisation 3D d'une paroi perforée, le terme d'inertie à travers les fentes épaisses est directement calculé par le modèle. Une perte de charge singulière est ajoutée à chaque changement de section pour égaliser la perte de charge singulière théorique, soit :

avec $K^{\prime}=\frac{1}{k_{c}^{2}}$.

$$
\Delta p=\frac{1}{2} \rho K^{\prime}\left|\mathrm{v}_{\mathrm{d}}\right| \mathrm{v}_{\mathrm{d}}
$$

Les résultats de la comparaison sont présentés ci-dessous pour une amplitude incidente $\mathrm{a}_{\mathrm{i}}=16 \mathrm{~mm}$. Les deux méthodes montrent des coefficients de réflexion similaires, validant la modélisation 3D d'une paroi perforée fine ou épaisse.

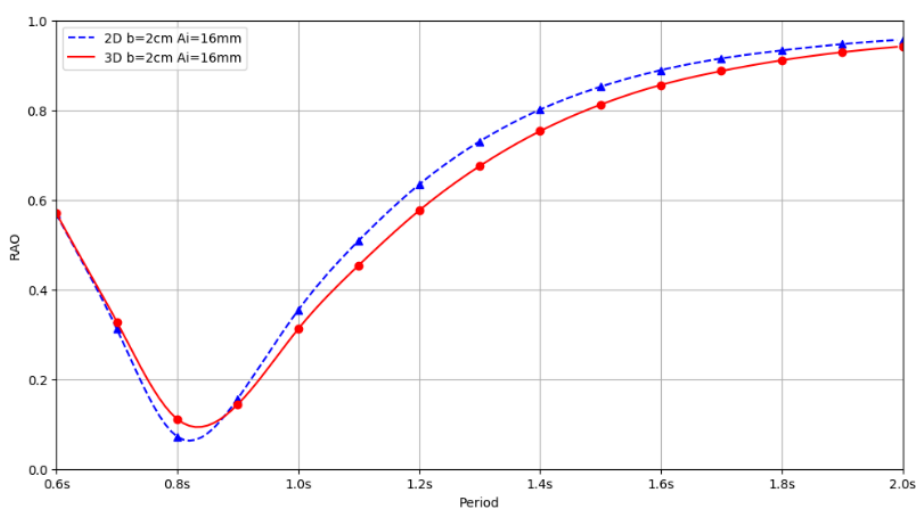

Figure 10. Coefficient de réflexion

Formulation théorique (2D) d'une paroi poreuse en bleu vs modélisation $3 D$ en rouge.

\section{Résultats expérimentaux et numériques : Gabions poreux espacés}

Les coefficients de réflexion expérimentaux (en bleu) sont comparés sur la figure 11 avec les coefficients de réflexion calculés numériquement (en rouge) sur une configuration de gabions poreux espacés créant des fentes épaisses. La courbe en transparence reporte les coefficients de réflexion numériques si les gabions espacés étaient des blocs solides, sans porosité.

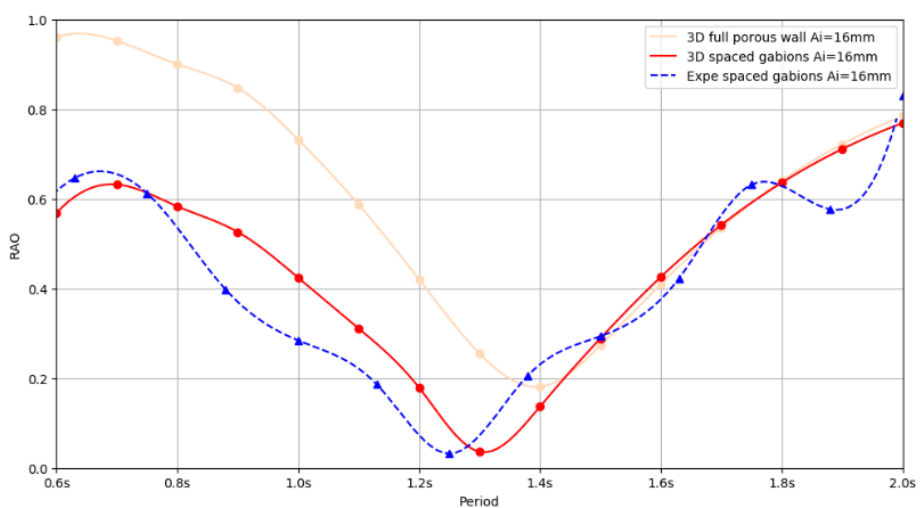

Figure 11. Coefficient de réflexion. 
Il y a une bonne correspondance entre les résultats expérimentaux et numériques, bien que les pics de performance ne se superposent pas tout à fait. L'incorporation de gabions à la place d'une structure pleine améliore la performance en aplatissant un petit peu la courbe de réflexion. Cela provoque aussi un décalage du pic vers les plus petites périodes. Le terme d'inertie de perte de pression est moins important.

\section{Application : enrochements et caisson Jarlan}

On a montré que l'outil numérique permet d'apprécier l'efficacité d'ouvrages de conception originale associant par exemple des blocs verticaux poreux en enrochements, plus ou moins espacés, de façon à créer de nouvelles pertes de charge qui s'ajouteraient à celles déjà présentes dans un milieu poreux simple supposé homogène.

L'installation d'enrochements à l'intérieur d'un caisson Jarlan est une autre application. Elle permet de jouer sur le comportement hydrodynamique du caisson tout en apportant du lest et des zones propices au développement des juvéniles.

Les exemples de configurations testées sont schématisées ci-dessous. Les configurations 1 et 2 correspondent à des caissons Jarlan classiques de longueurs de chambre respectives $3.70 \mathrm{~m}$ et $1.85 \mathrm{~m}$. Dans les configurations 3 et 4 , on place un milieu poreux sur $1.85 \mathrm{~m}$ de longueur et on fait évoluer la taille des blocs mis en place.

Les courbes ci-dessous indiquent l'évolution du coefficient de réflexion avec la période pour les différentes configurations décrites précédemment.

Le pic de performance d'un caisson Jarlan se situe lorsque $0.10<B / \lambda<0.15$. La mise en place d'enrochements à l'intérieur de la chambre permet de moduler le fonctionnement de la chambre Jarlan. On montre que la période optimale de dissipation se déplace entre les deux périodes associées à la longueur de chambre sans enrochements.

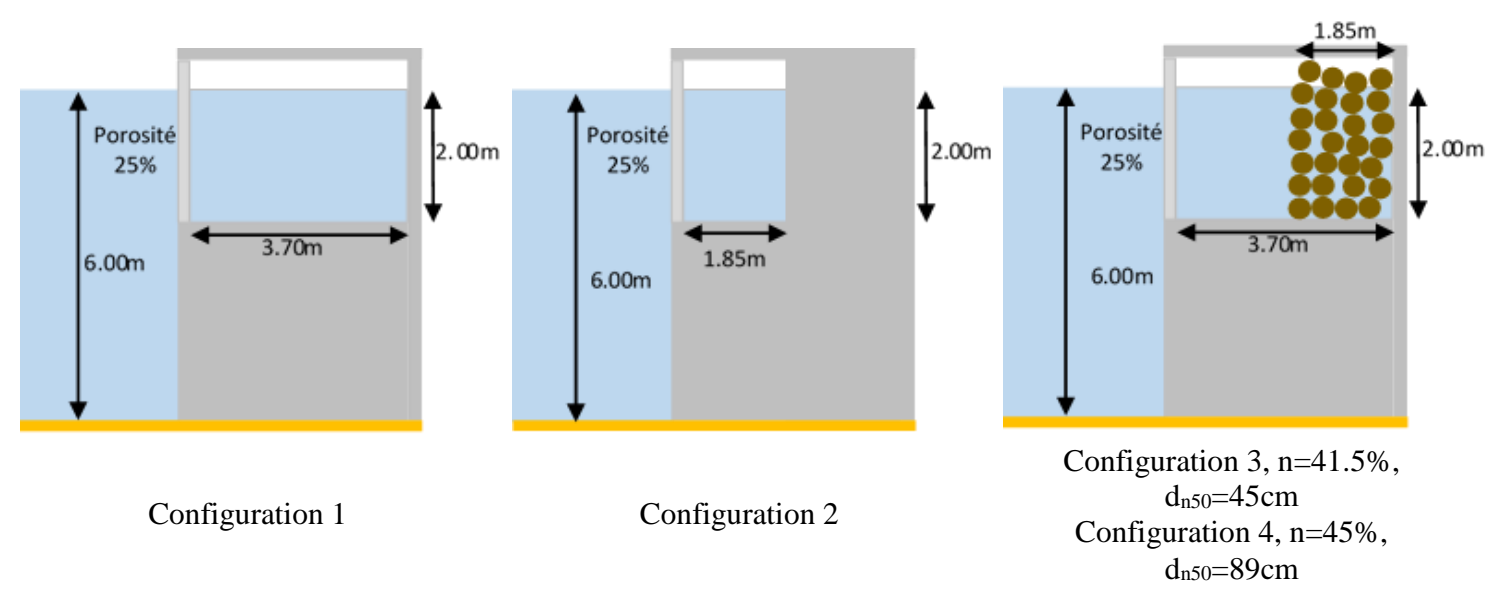

Figure 12. Schémas des configurations testées. 


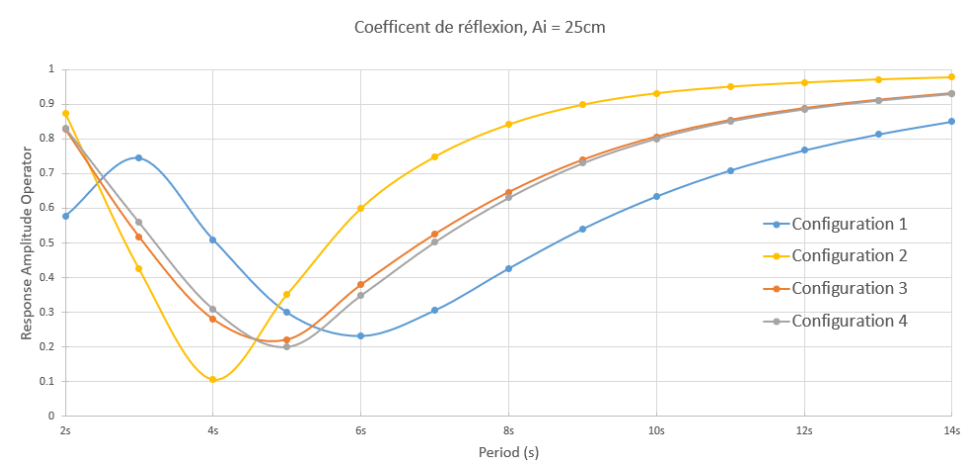

Figure 13. Efficacité des différentes configurations.

\section{Conclusion}

Les ouvrages amortisseurs en enrochements verticalisés sont des structures de conception innovantes qui permettent de respecter les herbiers en limitant l'impact au sol tout en apportant une protection supplémentaire aux bassins portuaires comme c'est le cas pour le port de Banyuls-sur-Mer. Ils s'associent facilement à d'autres ouvrages plus classiques tels que les caissons Jarlan. L'outil numérique développé doit permettre de cerner les meilleures compositions possibles entre enrochements et ouvrages classiques pour améliorer les conceptions originales alliant contraintes techniques et environnementales.

\section{Références}

ANDERSEN T.L., ELDRUP M.R., FRIGAARD P. (2017). Estimation of incident and reflected components in highly nonlinear regular waves. Coastal Engineering, Vol. 119, pp 51-54. https://doi.org/10.1016/j.coastaleng.2016.08.013

BURCHARTH H.F., ANDERSEN O.H. (1995). On the one-dimensional steady and unsteady porous flow equation. Coastal Engineering, Vol. 24, pp 233-257. https://doi.org/10.1016/0378-3839(94)00025-S

FORCHHEIMER P. (1901). Wasserbewegung durch Boden, Z. Ver. Deutsch. Ing. 45, $1782-1788$.

MELLINK B. (2012). Numerical and experimental research of wave interaction with a porous breakwater. Thesis, Delft University of Technology.

LAJOIE D. (2008). Optimisation du fonctionnement des atténuateurs de houle de type "dos de chameau" à l'aide de perforations dans la structure. $\mathrm{X}^{\mathrm{èmes}}$ JNGCGC, Sophia Antipolis, pp 749-760. http://dx.doi.org/10.5150/jngcgc.2008.071-L

POLUBARINOVA-KOCHINA P.Y. (1952). Theory of ground water movement. Gostekhizdat Moscow (in russian), English transl. by R.J.M. DE WIEST, (1962), Princeton University Press.

SOLLIT C.K., CROSS R.H. (1972). Wave transmission through permeable breakwaters, $13^{\text {th }}$ Coast. Eng. Conf., Vol. 3, ASCE, Vancouver, pp 1827-1846. https://doi.org/10.9753/icce.v13.99 VAN GENT M.R.A. (1995). Wave interaction with permeable coastal structures. Thesis, Delft University of Technology. 